\title{
The Relationship Between Serum Pentraxine 3 Levels and Hematological Markers in Patients With Rheumatoid Arthritis
}

\author{
Mustafa Serdar SAĞ, ${ }^{1}$ Sinem SAĞ, ${ }^{1}$ İbrahim TEKEOĞLU, ${ }^{1}$ Ayhan KAMANLI, ${ }^{1}$ Kemal NAS, ${ }^{1}$ Halil HARMAN ${ }^{2}$ \\ ${ }^{1}$ Divison of Rheumatology, Department of Pysical Medicine and Rehabilitation, Sakarya University Faculty of Medicine, Sakarya, Turkey \\ ${ }^{2}$ Divison of Rheumatology, Department of Pysical Medicine and Rehabilitation, Abant İzzet Baysal University Faculty of Medicine, Bolu, Turkey
}

\begin{abstract}
Objectives: This study aims to investigate the association of pentraxin 3 (PTX3) with neutrophil/lymphocyte ratio (NLR) rather than the disease activity score 28 using C-reactive protein in rheumatoid arthritis (RA).

Patients and methods: The study included 59 RA patients (11 males, 48 females; mean age $53.79 \pm 13.55$ years; range 40 to 66 years) and 20 healthy controls ( 5 males, 15 females; mean age $50.41 \pm 6.11$ years; range 43 to 56 years). Complete blood count tests were recorded and NLR and platelet/lymphocyte ratio were calculated. PTX3 and interleukin- 6 levels were examined in serum samples. Disease activity of RA patients was assessed by disease activity score 28.

Results: Demographic characteristics were similar between the two groups, with no statistically significant difference in terms of sex, age, and body mass index ( $p>0.05)$. NLR, PTX3 and interleukin- 6 levels were higher in patients with RA than the control group ( $p<0.05$ ). While erythrocyte sedimentation rate had a positive correlation with mean platelet volume, we found no correlation between NLR and other parameters of disease activity, PTX3, and interleukin-6.

Conclusion: We found no correlation between PTX3 and disease activity score 28 or NLR, although PTX3 levels were higher in RA patients than the controls. As a result, we were unable to establish a relationship between PTX3 and disease activity, directly or indirectly. To our knowledge, our study was the first to investigate the relationship between PTX3 and NLR.

Keywords: Neutrophil-lymphocyte ratio; pentraxin-3; rheumatoid arthritis.
\end{abstract}

Rheumatoid arthritis (RA) is a chronic disease that causes the activation of proinflammatory pathways leading to joint and systemic inflammation, and whose etiology is still unclear. ${ }^{1}$

Inflammation is the main determinant and basic mechanism that leads to disability and increased mortality rates in RA. ${ }^{2}$ Erythrocyte sedimentation rate (ESR) and C-reactive protein (CRP) are most frequently used to determine the level of inflammation in clinical practice. ${ }^{3}$ However, both of these markers have some limitations, such as short-term reflectance of inflammatory activity and being elevated in other infectious and inflammatory conditions. ${ }^{4,5}$

The neutrophil-lymphocyte ratio (NLR) is the ratio of the absolute neutrophil count to the lymphocyte count and this ratio is obtained from the complete blood count test. NLR has been shown to be beneficial in assessing inflammatory activity in chronic inflammatory diseases such as ulcerative colitis and familial Mediterranean fever. ${ }^{6,7}$ In recent years, it was shown that these markers may be associated with disease activity in RA as well. ${ }^{8-10}$

Pentraxins (PTXs) are separated into two groups, short and long PTXs. While PTX3 is a prototypical long PTX, acute phase reactants CRP and serum amyloid P are short PTXs. ${ }^{11}$ CRP is produced in the liver in response to interleukin-6 (IL-6) and is a systemic inflammatory response marker. PTX3 is produced by smooth muscle cells as well as innate immunity cells such 
as neutrophils, fibroblasts, epithelial cells, and vascular endothelial cells. In the literature, serum PTX3 levels were found to be high in RA. ${ }^{12,13}$

Despite the high levels of PTX in RA patients, no direct correlation was detected between PTX and disease activity. In contrast, CRP was associated with disease activity. ${ }^{3}$ This may be due to the use of CRP in disease activity score 28 (DAS28). NRL has also been associated with disease activity in RA. ${ }^{8-10}$ Therefore, in this study, we aimed to investigate the association of PTX3 with NLR rather than the DAS28 disease activity score using CRP in RA.

\section{PATIENTS AND METHODS}

This study was conducted between July 2015 and March 2016 at Sakarya University Faculty of Medicine. The study protocol was approved by the Sakarya University Faculty of Medicine Ethics Committee. A written informed consent was obtained from each patient. The study was conducted in accordance with the principles of the Declaration of Helsinki.

The study included 59 RA patients (11 males, 48 females; mean age $53.79 \pm 13.55$ years; range 40 to 66 years) who were diagnosed according to the American College of Rheumatology/ European League Against Rheumatism 2010 RA classification criteria and 20 healthy controls (5 males, 15 females; mean age 50.41 \pm 6.11 years; range 43 to 56 years). The control group consisted of age- and sex-matched healthcare personnel and patient accompanists without a known disease.

Those with acute-subacute infections, diabetes mellitus, chronic diseases such as cardiovascular disease, malignant hypertension, etc., inflammatory disease other than RA, such as ulcerative colitis, liver and kidney failure, hematological diseases, those who were pregnant and lactating, those under the age of 18 were excluded. The age, sex, age of illness, body mass index, medication usage, comorbidities, smoking status of the patients were recorded.

The disease activity of RA patients was assessed by DAS28. A score of DAS28 between 2.6 and 3.2 indicates low, 3.2-5.1 indicates moderate, and $>5.1$ indicates high disease activity. ${ }^{14}$
The venous blood of each patient was taken from the antecubital area in the morning after an overnight fast. Venous blood was drawn into tubes with or without anticoagulant. Standard complete blood count, liver and kidney function tests, ESR, CRP, rheumatoid factor, and anticyclic citrullinated peptide antibody tests were studied in the hospital's central laboratory. Neutrophil, lymphocyte, platelet, platelet distribution width, and mean platelet volume (MPV) values were recorded in complete blood count. NLR and platelet/lymphocyte ratio were calculated with a calculator. All the above parameters were evaluated and calculated using a Cell-Dyn 3700 SL hematology analyzer (Abbott, Chicago, IL, USA). ESR was measured immediately after blood collection using a Greiner SRS 20/II instrument (Vacuette Greiner, Kremsmünster, Austria). Rheumatoid factor and CRP levels were measured by nephelometric methods, using an IMAGE 800 analyzer (Beckman Coulter Inc., Brea, CA, USA). Anticyclic citrullinated peptide antibodies were measured via enzyme-linked immunosorbent assay (ELISA) and a result was considered positive if the level was above a cutoff of five arbitrary units (as suggested by Abbott ARCHITECT i1000SR).

Pentraxin 3 serum level was determined quantitatively by ELISA with an ELISA microplate strip washer (ELX50; BioTek Instruments, Winooski, VT, USA) and ELISA microplate reader (Biotek Instruments ELX800 Absorbance Reader, Winooski, VT, USA). PTX3 was studied using a commercially available kit (Boster Immunoleader, Fremont, CA, USA). We measured IL-6 concentrations using a solid-phase sandwich ELISA (Boster Immunoleader, Fremont, CA, USA). The sensitivity of these tests is $<10 \mathrm{pg} / \mathrm{mL}$, $<10 \mathrm{pg} / \mathrm{mL}$, and $<0.3 \mathrm{pg} / \mathrm{mL}$, respectively.

\section{Statistical analysis}

The obtained data were analyzed by SPSS for Windows version 16.0 (SPSS Inc., Chicago, IL, USA) statistical package program. In the comparison of the means of the groups, the student t-test was used for the normally distributed data, and Mann-Whitney $U$ test and KruskalWallis test were used for the data with no normal distribution. The Chi-square test was employed for intergroup comparison of categorical variables. The Fischer exact Chi-square test was used 


\begin{tabular}{|c|c|c|c|c|c|c|c|}
\hline \multirow[t]{2}{*}{ Characteristics } & \multicolumn{3}{|c|}{ RA patients $(n=59)$} & \multicolumn{3}{|c|}{ Controls $(\mathrm{n}=20)$} & \multirow[b]{2}{*}{$p$} \\
\hline & $\mathrm{n}$ & $\%$ & Mean \pm SD & $\mathrm{n}$ & $\%$ & Mean \pm SD & \\
\hline Age (year) & & & $53.8 \pm 13.6$ & & & $50.4 \pm 6.1$ & $>0.05^{*}$ \\
\hline Body mass index $\left(\mathrm{kg} / \mathrm{m}^{2}\right)$ & & & $28.4 \pm 3.5$ & & & $27.3 \pm 1.9$ & $>0.05^{*}$ \\
\hline \multicolumn{8}{|l|}{ Sex } \\
\hline Females & 48 & 81.4 & & 15 & 75.0 & & $>0.05 \neq$ \\
\hline Males & 11 & 12.1 & & 5 & 25.0 & & \\
\hline
\end{tabular}

when the expected value in the $2 \times 2$ tables was below 5 and the data were expressed as number and percentage. Spearman's correlation analysis was used to evaluate the inter-parameter relations. The results were assessed at a confidence interval of $95 \%$ and at a significance level of 0.05 .

\section{RESULTS}

Demographic characteristics were similar between RA and control groups, with no statistically significant difference in terms of sex, age, and body mass index ( $>>0.05)$ (Table 1).

Mean duration of illness of RA patients was 7.6 \pm 6.6 years, meanESR was $23.6 \pm 13.1 \mathrm{~mm} /$ hour, mean CRP was $7.4 \pm 5.9 \mathrm{mg} / \mathrm{dL}$, and mean DAS28 was $3.1 \pm 1.2$. Rheumatoid factor positivity rate was $55.1 \%$ and the anticyclic citrullinated peptide positivity rate was $66.1 \%$ in the RA group. Smoking rates in the RA and control groups were $41.4 \%$ and $35 \%$, respectively.

White blood cells, NLR, PTX3, and IL-6 levels were significantly higher in the RA group than the control group (Table 2). While a significant positive correlation was found between MPV and ESR and DAS28-CRP, no significant correlation was found between the other parameters (Table 3).

\section{DISCUSSION}

In this study, we found that NLR, PTX3, and IL-6 levels in RA patients were higher than the control group. While ESR had a positive correlation with MPV, we found no correlation between NLR and other parameters of disease activity, PTX3 and IL-6. Systemic inflammation is associated with changes in the amount and composition of circulating blood cells. Normochromic anemia, thrombocytosis, neutrophilia, and lymphopenia may often be observed with many inflammatory conditions. ${ }^{15}$ Therefore, the properties of components of circulating blood cells may be used to assess inflammatory activity. ${ }^{16}$ Neutrophils release proteases, prostaglandins, and reactive oxygen radicals into the synovial space in patients with active RA. In addition, B-lymphocyte stimulator has the potential to exacerbate the disease by activating other cells through tumor

Table 2. Comparison of laboratory parameters of study groups

\begin{tabular}{lcccc}
\hline & RA patients $(\mathrm{n}=59)$ & & Controls $(\mathrm{n}=20)$ & \\
\cline { 2 - 2 } & Mean $\pm \mathrm{SD}$ & & Mean $\pm \mathrm{SD}$ & \\
\hline White blood cell & $8.1 \pm 2.6$ & & $6.6 \pm 1.2$ & $0.020^{*}$ \\
Platelet $\left(\times 10^{3} / \mu \mathrm{L}\right)$ & $279.9 \pm 83.6$ & & $278.1 \pm 51.0$ & 0.808 \\
Mean platelet volume $(\mathrm{fl})$ & $7.5 \pm 1.1$ & & $7.6 \pm 1.7$ & 0.664 \\
Platelet distribution width $(\%)$ & $17.5 \pm 0.9$ & & $18.1 \pm 1.9$ & 0.467 \\
Neutrophil $(\mu \mathrm{L})$ & $5.0 \pm 2.0$ & & $3.8 \pm 0.9$ & 0.018 \\
Lymphocyte $(\mu \mathrm{L})$ & $2.2 \pm 1.0$ & & $2.2 \pm 0.6$ & 0.318 \\
Neutrophil/lymphocyte ratio & $2.5 \pm 1.1$ & & $1.8 \pm 0.7$ & 0.014 \\
Platelet/lymphocyte ratio & $142.2 \pm 58.8$ & & $134.3 \pm 51.0$ & 0.457 \\
Pentraxin-3 $(\mathrm{ng} / \mathrm{mL})$ & $709.8 \pm 575.1$ & $363.1 \pm 88.4$ & 0.000 \\
Interleukin-6 $(\mathrm{pg} / \mathrm{mL})$ & $14.8 \pm 11.1$ & $13.5 \pm 2.4$ & $0.028^{*}$ \\
\hline RA: Rheumatoid arthritis; SD: Standard deviation; Mann-Whitney U test; ${ }^{*} \mathrm{p}<0.05$. & \\
\hline
\end{tabular}




\begin{tabular}{|c|c|c|c|c|c|}
\hline & РTX3 & IL-6 & ESR & CRP & DAS28 \\
\hline & $\mathrm{r}$ & $\mathrm{r}$ & $\mathrm{r}$ & $\mathrm{r}$ & $\mathrm{r}$ \\
\hline Pentraxin & - & 0.025 & 0.013 & 0.098 & 0.001 \\
\hline Neutrophil/lymphocyte ratio & 0.179 & 0.105 & 0.069 & 0.009 & $0.273^{*}$ \\
\hline Platelet/lymphocyte ratio & 0.039 & 0.166 & 0.036 & 0.149 & 0.070 \\
\hline Mean platelet volume & 0.063 & 0.172 & $0.362^{*}$ & 0.094 & -0.099 \\
\hline Platelet distribution width & 0.130 & 0.149 & 0.244 & 0.046 & -0.042 \\
\hline Platelet & 0.059 & 0.114 & 0.113 & 0.140 & 0.210 \\
\hline Disease activity score 28 & 0.001 & 0.239 & 0.149 & $0.326^{*}$ & \\
\hline
\end{tabular}

necrosis factor and numerous other mediator secretions. ${ }^{1,17,18}$

Neutrophil/lymphocyte ratio is a routine marker of neutrophil and lymphocyte levels associated with complete blood count. It has been shown to be useful in the evaluation of systemic inflammation in a variety of diseases such as coronary artery disease, diabetes mellitus, Alzheimer's disease, ulcerative colitis, and cancer. ${ }^{6,19-22}$ Additionally, NLR has been found to have a prognostic value in cardiovascular diseases and in some types of cancer, independent of other factors. ${ }^{22,23}$ NLR has been shown to be higher in RA group compared to control group and associated with disease activity in several publications. ${ }^{8-10}$ We also found that NLR was higher in RA patients than the control group and a positive significant correlation was present with DAS28.

In the active stage of RA, increased platelet count may be observed; the platelet count may decrease with the remission of inflammation. However, there is not enough information about the detailed roles of platelets. The platelet count, MPV and platelet distribution width are the three useful indices of platelet function that reflect platelet production rate and stimulation of this production. ${ }^{24}$ Studies have shown that MPV and platelet distribution width are both associated with disease activity in RA. ${ }^{25,26}$ Kisacik et al. ${ }^{16}$ have shown that the MPV values in patients with ankylosing spondylitis and RA were significantly lower than controls and there was a significant negative correlation between the MPV value and the level of disease activity. The study of Tekeoglu et al. ${ }^{8}$ supports this study and found a negative correlation between MPV values and disease activity. Yazici et al. $^{25}$ found that the MPV values in RA patients were positively correlated with both DAS28 and disease activity levels. In our study, we found a significant positive correlation between the MPV value and the disease activity parameter, ESR.

Pentraxin 3 is produced by smooth muscle cells as well as innate immunity cells such as neutrophils, fibroblasts, epithelial cells, and vascular endothelial cells. High concentrations of PTX3 were found in autoimmune and degenerative disorders. ${ }^{12,13}$

Although PTX3 levels have been studied in various rheumatic and autoimmune diseases and levels of PTX3 have been correlated with disease activity and response to therapy, its role in systemic autoimmune disease is not well understood. ${ }^{27,28} \mathrm{In}$ a study of Satomura et al., ${ }^{29}$ serum amyloid A has been shown to initiate the production of another proinflammatory molecule, PTX3, in rheumatoid synovitis. PTX3 triggered by serum amyloid A production has been expressed to be included in the activation of inflammatory cells and tissue damage in the rheumatoid synovium. Recent studies have shown that PTX3 levels are higher in patients with RA than controls. ${ }^{30}$ However, there was no correlation between PTX3 and DAS28. We also found that the PTX3 level in the RA group was higher than the control group. We did not find a significant relationship between the PTX3 level and the disease activity parameters, ESR, CRP, and DAS28. In addition, PTX3 level did not correlate with hematological markers associated with inflammation such as NLR and platelet/lymphocyte ratio. 
We had patients with clinically high disease activity, even though their ESR and CRP values were within normal limits. These patients may have affected correlations of hematological markers with disease activity and PTX3.

Most of the inflammatory processes involved in RA pathophysiology are regulated by cytokines. The imbalance between proand anti-inflammatory cytokines supports the onset of autoimmune, chronic inflammation and consequently joint damage. ${ }^{30}$ Of the proinflammatory cytokines, which play an important role in the pathogenesis of RA, IL-6 has been detected at high rates in the synovial fluid of RA patients and this elevation has been found to be correlated with disease activity and joint damage. ${ }^{31,32}$ In our study, IL-6 level was higher in RA group. However, we did not find any relationship with hematological markers and DAS28, ESR, or CRP.

Our study has some limitations. Firstly, the sample size was small. Secondly, the disease activity score was not balanced. Furthermore, hematological markers were assessed once crosssectionally. The venous blood values obtained with certain periods could give a healthier result. Future studies resolving these limitations may investigate the relationship between PTX3 and RA activity more substantially.

In conclusion, no correlation has been detected between PTX3 and disease activity in RA patients in previous studies. We predicted that this may be due to the use of CRP or ESR in the DAS28. We thought that there was no correlation between PTX3 and disease activity because there was no positive correlation between PTX3 and CRP and ESR. Therefore, we investigated the relationship between PTX3 and NLR, which was related to disease activity in studies conducted. Thus, we assessed whether there was an indirect link between PTX3 and disease activity. However, we found no correlation between PTX3 and DAS28 or NLR, although PTX3 were found to be higher in patients with RA than the control group. We could not determine any relationship between PTX3 and disease activity. To our knowledge, our study was the first to investigate the relationship between PTX3 and NLR.

\section{Declaration of conflicting interests}

The authors declared no conflicts of interest with respect to the authorship and/or publication of this article.

\section{Funding}

The authors received no financial support for the research and/or authorship of this article.

\section{REFERENCES}

1. McInnes IB, Schett $G$. The pathogenesis of rheumatoid arthritis. N Engl J Med 2011;365:2205-19.

2. Zochling J, Braun J. Mortality in rheumatoid arthritis and ankylosing spondylitis. Clin Exp Rheumatol 2009;27:127-30.

3. Hobbs KF, Cohen MD. Rheumatoid arthritis disease measurement: a new old idea. Rheumatology (Oxford) 2012;51:21-7.

4. Colglazier CL, Sutej PG. Laboratory testing in the rheumatic diseases: a practical review. South Med J 2005;98:185-91.

5. Kavanaugh A. The role of the laboratory in the evaluation of rheumatic diseases. Clin Cornerstone 1999;2:11-25.

6. Torun S, Tunc BD, Suvak B, Yildiz H, Tas A, Sayilir $A$, et al. Assessment of neutrophil-lymphocyte ratio in ulcerative colitis: a promising marker in predicting disease severity. Clin Res Hepatol Gastroenterol 2012;36:491-7.

7. Ahsen A, Ulu MS, Yuksel S, Demir K, Uysal M, Erdogan M, et al. As a new inflammatory marker for familial Mediterranean fever: neutrophil-tolymphocyte ratio. Inflammation 2013;36:1357-62.

8. Tekeoğlu İ, Gürol G, Harman H, Karakeçe E, Çiftçi IH. Overlooked hematological markers of disease activity in rheumatoid arthritis. Int $J$ Rheum Dis 2016;19:1078-82.

9. Mercan R, Bitik B, Tufan A, Bozbulut UB, Atas $\mathrm{N}$, Ozturk MA, et al. The Association Between Neutrophil/Lymphocyte Ratio and Disease Activity in Rheumatoid Arthritis and Ankylosing Spondylitis. J Clin Lab Anal 2016;30:597-601.

10. Siloși I, Boldeanu MV, Cojocaru M, Biciușca V, Padureanu V, Bogdan $\mathrm{M}$, et al. The Relationship of Cytokines IL-13 and IL-17 with Autoantibodies Profile in Early Rheumatoid Arthritis. J Immunol Res 2016;2016:3109135.

11. Garlanda C, Bottazzi B, Bastone A, Mantovani A. Pentraxins at the crossroads between innate immunity, inflammation, matrix deposition, and female fertility. Annu Rev Immunol 2005;23:337-66.

12. Knoflach M, Kiechl S, Mantovani A, Cuccovillo I, Bottazzi B, Xu Q, et al. Pentraxin-3 as a marker of advanced atherosclerosis results from the Bruneck, ARMY and ARFY Studies. PLoS One 2012;7:31474. 
13. Wang H, Wang K, Wang C, Zhong X, Qiu W, Hu X. Increased plasma levels of pentraxin 3 in patients with multiple sclerosis and neuromyelitis optica. Mult Scler 2013;19:926-31.

14. Wells G, Becker JC, Teng J, Dougados M, Schiff M, Smolen J, et al. Validation of the 28-joint Disease Activity Score (DAS28) and European League Against Rheumatism response criteria based on C-reactive protein against disease progression in patients with rheumatoid arthritis, and comparison with the DAS28 based on erythrocyte sedimentation rate. Ann Rheum Dis 2009;68:954-60.

15. Gabay C, Kushner I. Acute-phase proteins and other systemic responses to inflammation. $\mathrm{N}$ Engl J Med 1999;340:448-54.

16. Kisacik B, Tufan A, Kalyoncu U, Karadag O, Akdogan A, Ozturk MA, et al. Mean platelet volume (MPV) as an inflammatory marker in ankylosing spondylitis and rheumatoid arthritis. Joint Bone Spine 2008;75:291-4.

17. Kouri VP, Olkkonen J, Ainola M, Li TF, Björkman L, Konttinen YT, et al. Neutrophils produce interleukin$17 \mathrm{~B}$ in rheumatoid synovial tissue. Rheumatology (Oxford) 2014;53:39-47.

18. Cascão R, Rosário HS, Souto-Carneiro MM, Fonseca JE. Neutrophils in rheumatoid arthritis: More than simple final effectors. Autoimmun Rev 2010;9:531-5.

19. Tamhane UU, Aneja S, Montgomery D, Rogers EK, Eagle KA, Gurm HS. Association between admission neutrophil to lymphocyte ratio and outcomes in patients with acute coronary syndrome. Am J Cardiol 2008;102:653-7.

20. Öztürk ZA, Kuyumcu ME, Yesil Y, Savas E, Ylldız H, Kepekçi $Y$, et al. Is there a link between neutrophillymphocyte ratio and microvascular complications in geriatric diabetic patients? J Endocrinol Invest 2013;36:593-9.

21. Kuyumcu ME, Yesil Y, Oztürk ZA, Kizilarslanoğlu C, Etgül S, Halil M, et al. The evaluation of neutrophillymphocyte ratio in Alzheimer's disease. Dement Geriatr Cogn Disord 2012;34:69-74.

22. Fowler AJ, Agha RA. Neutrophil/lymphocyte ratio is related to the severity of coronary artery disease and clinical outcome in patients undergoing angiography-the growing versatility of NLR. Atherosclerosis 2013;228:44-5.

23. Proctor MJ, McMillan DC, Morrison DS, Fletcher CD, Horgan PG, Clarke SJ. A derived neutrophil to lymphocyte ratio predicts survival in patients with cancer. Br J Cancer 2012;107:695-9.

24. Briggs C. Quality counts: new parameters in blood cell counting. Int J Lab Hematol 2009;31:277-97.

25. Yazici S, Yazici M, Erer B, Erer B, Calik Y, Ozhan H, et al. The platelet indices in patients with rheumatoid arthritis: mean platelet volume reflects disease activity. Platelets 2010;21:122-5.

26. Mahmoud Muddathir AR, Haj FEJ. Platelet indices in sudanese patients with rheumatoid arthritis. Asian $\mathrm{J}$ Biomed Pharm Sci 2013;3:1-3.

27. Luchetti MM, Piccinini G, Mantovani A, Peri G, Matteucci C, Pomponio G, et al. Expression and production of the long pentraxin PTX3 in rheumatoid arthritis (RA). Clin Exp Immunol 2000;119:196-202.

28. Luchetti MM, Sambo P, Majlingová P, Svegliati Baroni S, Peri G, Paroncini P, et al. Scleroderma fibroblasts constitutively express the long pentraxin PTX3. Clin Exp Rheumatol 2004;22:66-72.

29. Satomura K, Torigoshi T, Koga T, Maeda Y, Izumi $\mathrm{Y}$, Jiuchi $\mathrm{Y}$, et al. Serum amyloid A (SAA) induces pentraxin 3 (PTX3) production in rheumatoid synoviocytes. Mod Rheumatol 2013;23:28-35.

30. Tekeoğlu İ, Harman H, Sağ S, Altındiğ M, Kamanlı A, Nas K. Levels of serum pentraxin 3, IL-6, fetuin A and insulin in patients with rheumatoid arthritis. Cytokine 2016;83:171-5.

31. Hirano T, Matsuda T, Turner M, Miyasaka N, Buchan $G$, Tang $B$, et al. Excessive production of interleukin 6/B cell stimulatory factor- 2 in rheumatoid arthritis. Eur J Immunol 1988;18:1797-801.

32. Baillet A, Gossec L, Paternotte S, Etcheto A, Combe $\mathrm{B}$, Meyer $\mathrm{O}$, et al. Evaluation of serum interleukin- 6 level as a surrogate marker of synovial inflammation and as a factor of structural progression in early rheumatoid arthritis: results from a French national multicenter cohort. Arthritis Care Res (Hoboken) 2015;67:905-12. 